\title{
The application of multi-frequency fringe projection profilometry on the measurement of biological tissues
}

\author{
Chao Jiang ${ }^{\mathrm{a}}$, Shuhai Jia ${ }^{\mathrm{a},}$, Y Yong Xu ${ }^{\mathrm{a}}$, Qingchen Bao ${ }^{\mathrm{a}}$, Jun Dong ${ }^{\mathrm{a}}$ and Qin Lian ${ }^{\mathrm{a}, \mathrm{b}}$ \\ ${ }^{a}$ School of Mechanical Engineering, Xi'an Jiaotong University, Xi'an 710049, China \\ ${ }^{b}$ Shaanxi Hengtong Intelligent Machine Co., Ltd, Xi'an 710049, China
}

\begin{abstract}
A volume of research has been performed on the optical surface profilometry in the field of biomedicine and the optical system with the phase-measuring method becomes the main emphasis of the research. In this research, a brand new fringe projection profilometry with multiple frequencies is described for measuring the biological tissue. A pork liver, as an object, is regarded as a human organ and a DMD projector is used to generate the multi-frequency fringe patterns. The wrapped phase maps are obtained by means of the five-step phase shifting method and calculated via a peak searching algorithm in which the process of measuring the point on the surface of the object is independent so that the step of unwrapping the phase can be avoided. The final results given are acceptable which confirm this method and suggest its enormous potential for the biomedical measurements.
\end{abstract}

Keywords: Biomedicine measurements, fringe projection, phase shifting method, peak searching algorithm

\section{Introduction}

With the development of $3 \mathrm{D}$ representation technique, there is a need for accurately measuring the shape of objects in a variety of areas. In medicine, on the basis of the 3D representation technique, the study of complex structures such as bone structure, acetabular fractures and craniofacial abnormalities becomes reality [1-4]. Owing to the advantages of low cost, short time, and no invasion, 3D ultrasonic echo image is widely used in the measurement of organs. However, it cannot overcome the influence of the gas and the skeleton. Compared with 3D ultrasonic echo image, computed tomography (CT) provides higher accuracy, but the patient cannot be measured continuously within the radiation dose since the X-rays required is harmful to the human organs. Magnetic Resonance Imaging (MRI) is an alternative method for its advantage of multiple plane imaging. Nevertheless, it also brings the drawback of higher cost, no quantitative analysis and the need for a static posture during the long measuring time [5].

In recent years, the usage of the profilometry based on optics with no contact and large measuring range in the field of 3D surface of biological tissues has drawn more and more attention such as the

\footnotetext{
* Address for correspondence: Shuhai Jia, School of Mechanical Engineering, Xi'an Jiaotong University, Xi'an 710049, China. Tel.:13152068353; Fax: +86-029-82660487; E-mail: shjia@mail.xjtu.edu.cn.
} 
application of the 3D laser scanner in the soft-tissue evaluation [6]. Nevertheless, different from the harm from the high-power light like laser on the retina, the methods on the foundation of fringe projection have been widely discussed and applied to practice owing to their higher safety, better performance, higher resolution and simpler operation such as the usage of the optical acquisition system with the phase-measuring profilometry on the facial prostheses [7-9]. In a representative system of fringe projection profilometry, the structured fringe patterns are projected on the object using a DMD projector and the distorted fringes are recorded by a charge coupled device (CCD). Many methods such as PMP and FTP apply to the calculation of the phase information of the fringe pattern [10-12]. Compared with FTP, although more images are needed, the result of PMP is more accurate since the process of filtering in FTP is avoided. The choice of the different phase shifting techniques depends on the noise sources to be mitigated [13]. On account of the fact that the phase map is wrapped in the range of $-\pi-\pi$, one of the challenges is the phase unwrapping step. The methods such as reliability-guided phase unwrapping algorithm and fast transforms and iterative method provide solutions to solve the problem $[14,15]$. However, these methods are path-dependent and continuous phase-required. The process of correctly unwrapping the phase will be difficult if there are discontinuities on the object which depth is very large [16].

This present paper is aimed at describing a new method for measuring the surface of the biological tissues. Distinguished from the conventional fringe projection profilometry adopted in the reconstruction of the tissues, the phase information is achieved by five-step phase shifting method and the object height is calculated by a peak searching algorithm. Since the unwrapping process is avoided during the measurement, the new method is capable of measuring the objects with complex shapes, which provides benefits to the skin repair or the organ regeneration.

\section{Principles and methods}

A typical fringe projection profilometry system usually consists of a computer, a DMD projector, a reference plane and a CCD camera, as shown in Figure 1.

The fringe patterns with multiple frequencies generated by the computer are projected onto the object and collected by the CCD camera after they are deformed owing to the shape of the object. The reference plane supporting the object provides a reference height when calculating the true height of the object.

\subsection{Achieving the wrapped information on the basis of five-step phase shifting method}

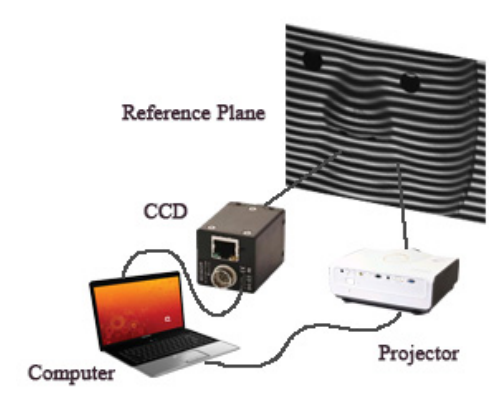

Fig. 1. The layout of the fringe projection profilometry.

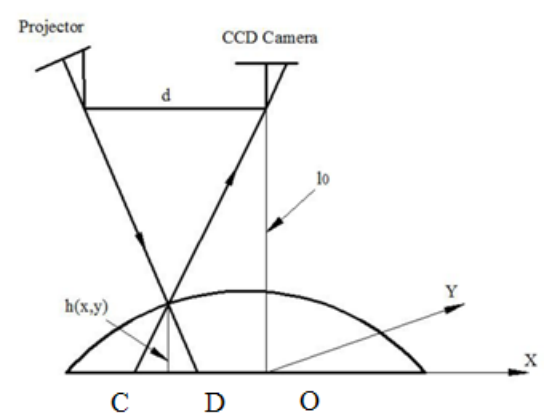

Fig. 2. The geometrical relationship of fringe projection technique. 
Figure 2 illustrates the geometrical relationship of the fringe projection profilometry. Suppose that $\mathrm{X}$ and $\mathrm{Y}$ are the horizontal and vertical directions of the reference plane respectively while $\mathrm{h}$ represents the height of one point on the surface of the object and d represents the optic center distance between the CCD and the DMD projector. D represents the position of a fringe pattern on the reference plane in the $\mathrm{X}$ direction while $\mathrm{C}$ is the equivalent position of the same fringe pattern reflected from the object surface. $\mathrm{O}$ in Figure 2 is the point which is intersected by the optics axis and the reference plane. The deformation of the fringe patterns will appear if an object is placed in the area of measurement. The fringe patterns on the surface of the object and the reference plane can be written as:

$$
\begin{gathered}
O(x, y)=I_{0}(x, y)+M(x, y) \cos \left[2 \pi f_{0} x+\varphi_{O}(x, y)\right] \\
I(x, y)=I_{0}(x, y)+M(x, y) \cos \left[2 \pi f_{0} x+\varphi_{I}(x, y)\right]
\end{gathered}
$$

Here $I_{0}(x, y)$ is the background intensity and $M(x, y)$ is the modulation of the fringe, $f_{0}$ represents the selected frequency, $\varphi_{O}$ is the phase influenced from the object height and $\varphi_{I}$ is the phase of the reference plane, $O(x, y)$ is the fringe pattern deformed and $I(x, y)$ is the fringe pattern reflected from the reference plane.

During the process of adopting the multiple step phase shifting method, high-frequency noise will emerge owing to the nonlinear response of the measuring system. The light intensity error of the system can be written as:

$$
d I=e_{0}+\left(e_{1}-1\right) I+e_{2} I^{2}+e_{3} I^{3}
$$

where $e_{i}(i=0,1,2,3)$ is the nonlinear coefficient of the order. After the usage of the $\mathrm{N}$ step phase shifting method, the relevant phase error $d \varphi$ takes the form as [17]:

$$
d \varphi=-\frac{2}{N}\left[\beta \sum_{i=0}^{N-1} \sin \left(3 \varphi+\frac{6 \pi i}{N}\right)+\gamma \sum_{i=0}^{N-1} \sin \left(4 \varphi+\frac{8 \pi i}{N}\right)\right]
$$

The parameters $\beta$ and $\gamma$ in Eq. (4) are defined as

$$
\begin{aligned}
& \beta=\frac{1}{4}\left(3 e_{3} I_{0} M+e_{2} M\right), \\
& \gamma=\frac{1}{8} e_{3} M .
\end{aligned}
$$

When $\mathrm{N}$ is equal to 4 ,

$$
d \varphi=-\frac{M^{2} e_{3}}{4} \sin (4 \varphi) .
$$


When $\mathrm{N}$ is equal or greater than 5 ,

$$
d \varphi=0
$$

Eqs. (6) and (7) show that when the step of the phase shifting method is 5 or greater than 5 , the phase error derived from the nonlinear response of the measuring system can be greatly reduced. Hence, the five-step phase shifting method is adopted to calculate the phase information of the object as well as the reference plane. Here, for illustrative purpose it is noted that the following expressions from five measurements of the reference plane can be formed with a $2 \pi / 5$ increment of the phase.

$$
\begin{aligned}
I_{i}(x, y) & =I_{0}(x, y)+M(x, y) \cos \left[2 \pi f_{0} x+\varphi_{I}(x, y)+\frac{2 \pi}{5} \times(i-1)\right](i=1,2,3,4,5) \\
\psi_{I} & =2 \pi f_{0} x+\varphi_{I}(x, y) \\
& =-\arctan \left[\frac{I_{1} \sin \frac{0 \pi}{5}+I_{2} \sin \frac{2 \pi}{5}+I_{3} \sin \frac{4 \pi}{5}+I_{4} \sin \frac{6 \pi}{5}+I_{5} \sin \frac{8 \pi}{5}}{I_{1} \cos \frac{0 \pi}{5}+I_{2} \cos \frac{2 \pi}{5}+I_{3} \cos \frac{4 \pi}{5}+I_{4} \cos \frac{6 \pi}{5}+I_{5} \cos \frac{8 \pi}{5}}\right]
\end{aligned}
$$

Similarly, the phase of the object can also be expressed in the same way. Then, the phase change derived from the object height can be written as

$$
\Delta \varphi(x, y)=\psi_{O}-\psi_{I}=\psi_{O}(x, y)-\psi_{I}(x, y) .
$$

According to Figure 2, it is noted that $\Delta \varphi(x, y)=2 \pi f_{0} \overline{C D}$, so the height of the object can be simplified to [18]:

$$
h(x, y)=\frac{l_{0} \Delta \varphi(x, y)}{2 \pi f_{0} d}
$$

in which $l_{0}$ represents the distance between the optic center of the CCD and the reference plane.

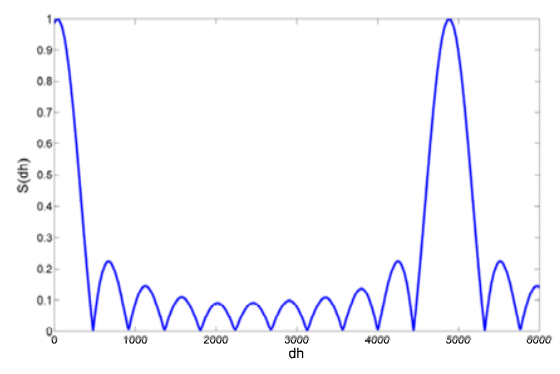

Fig. 3. The peak-searching curve. 


\subsection{Calculating the object height by peak-searching algorithm}

Since the sinusoid is periodical, a peak signal will be received by simply adding the sinusoids together and the high oscillation can be removed by taking advantage of the complex notation and the magnitude. Thus, this function takes the form as:

$$
S(d h)=\frac{1}{K}\left|\sum_{k=1}^{K} \exp \left\{\left[\frac{2 \pi f_{k} d}{l_{0}}(d h-h)\right] \bullet i\right\}\right|,
$$

where $d h$ is a variable which changes in a certain range in order to search the real height of the measured point. It can be noted from Eq. (12) that when the variable $d h$ is equal to $h$, the function will reach its main maximum. Thus, the real height of the object can be calculated by finding the value of variable $d h$ corresponding to the main maximum of the function $S(d h)$. The curve formed by $S(d h)$ is called the peak-searching curve which is illustrated in Figure 3.

Since the main maximum of $S(d h)$ is periodic and iterative, the range of the measurement (free height) is no more than the period of the peak-searching curve in order to guarantee the accuracy of the final result. If the frequencies of the fringe pattern selected are meeting the relationship as [17]:

$$
f_{k}=f_{k-1}+m
$$

the distance between the two highest peaks (free height) can be written as

$$
\text { FreeHeight }=\frac{l_{0}}{d m} \text {. }
$$

From Figure 2, the resolution of the measuring system is calculated as

$$
\text { resolution }=\frac{d s \bullet l_{0}}{l_{1}},
$$

where $d s$ is the pixel size of the CCD camera used, $l_{1}$ is the distance between the optic center and the target surface plane of the CCD camera and $l_{0}$ is the distance between the optic center of the CCD camera and the reference plane.

As was discussed above, the height of the object could be acquired on the foundation of the methods mentioned, in which the process of reconstructing the object is based on the phase information of the single point and has no requirements on its surrounding points, thus avoiding the trouble of unwrapping process and the problem of error accumulation existed in the conventional unwrapping methods, which guarantees the measurements of the objects with large depth discontinuities. 


\section{Experimental results}

\subsection{The comparison between the methods based on the simulated results}

For the sake of explaining the advantage of the peak-searching algorithm, a step was generated in the Matlab program in order to simulate the object with large discontinuities, as Figure 4(a) shows. Figures 4(b) and 4(c) illustrate the result of the reconstructed shape of the step by the peak-searching algorithm and the fast transforms and iterative method. Figure 4(d) shows the comparison between the results of the two methods adopted. From the figures, it can be seen that, compared with the fast transforms and iterative method, the peak-searching algorithm is more suitable for calculating the objects with large steps.

\subsection{The practical measurement on a pork liver}

In this study, a DMD projector was used to generate the fringe patterns. The fringe patterns reflected by the object were collected by a JAI GigE CCD camera which pixel size is $7.4 \mu m \times 7.4 \mu m$. The biological tissue was simulated by a pork liver. For the sake of meeting the demand from five-step phase shifting method, for one frequency, since the phase shifting for each step is $2 \pi / 5,10$ images were needed. The frequencies selected were in the range of $7 / 6$ fringes per $\mathrm{cm}-1.5$ fringes per $\mathrm{cm}$ at an interval of $1 / 30$ fringes per $\mathrm{cm}$. The parameter $\mathrm{d}$ in the actual measuring system is $9.023 \mathrm{~cm}, l_{0}$ is $70.376 \mathrm{~cm}$ and $l_{1}$ is $18.294 \mathrm{~cm}$.

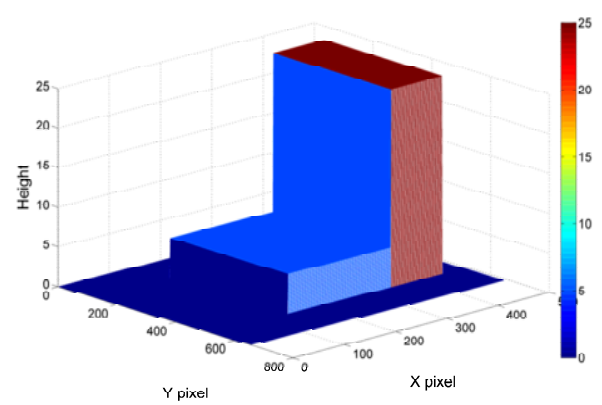

(a)

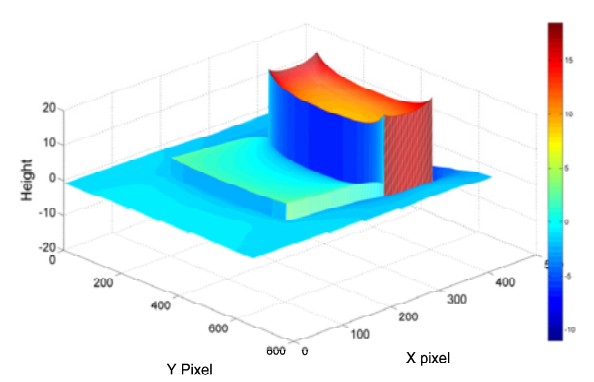

(c)

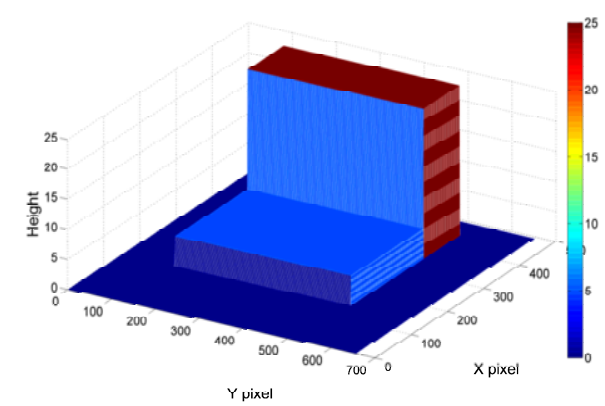

(b)

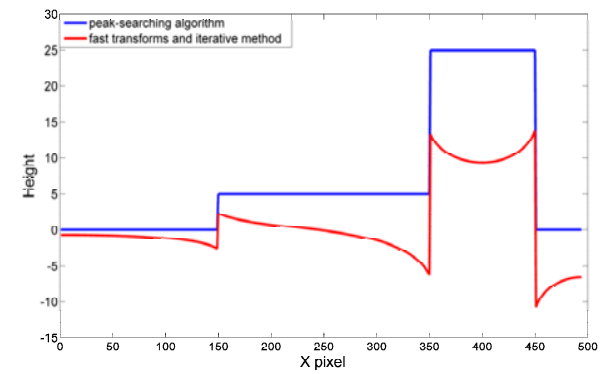

(d)

Fig. 4. (a) The step simulated to be measured. (b) The result of the reconstructed shape using the peak-searching algorithm. (c) The result of the reconstructed shape using fast transforms and iterative method. (d) The comparison between the peak-searching algorithm and the fast transforms and iterative method. 

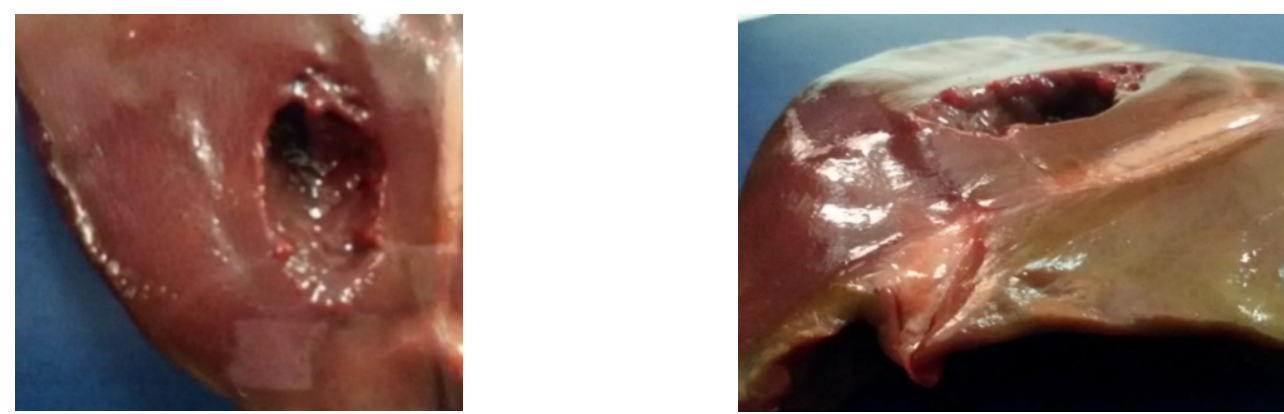

Fig. 5. The pork liver measured.

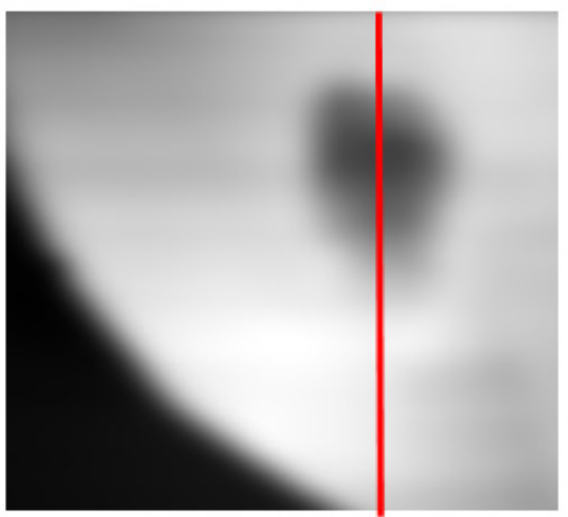

(a)

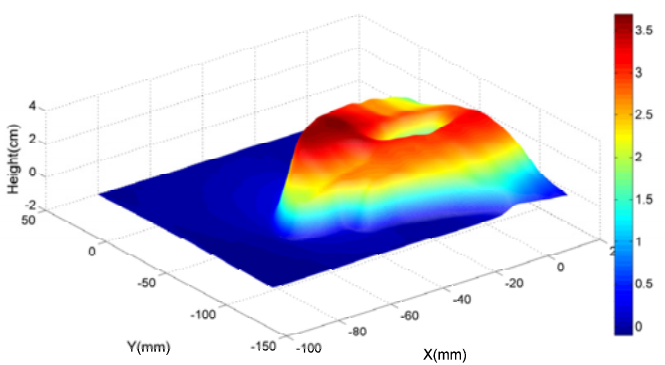

(b)

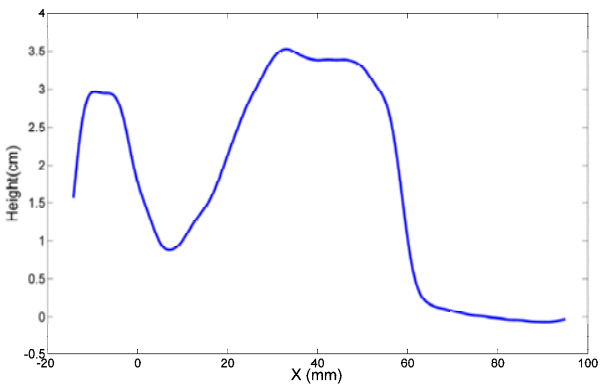

(c)

Fig. 6. (a) The gray map of the reconstructed 3D shape of the liver. (b) The reconstructed 3D shape of the liver with a hole measured. (c) The height distribution of the section marked with a red line in (a).

The pork livers measured in the experiment are displayed in Figure 5. The hole dug in the pork liver is used to simulate the removed pathological part of the human organ. The gray map and the reconstructed 3D shape of the measured liver are illustrated in Figure 6.

The sizes along the length, width and height of the pork liver are calibrated on the basis of Matlab calibration toolbox which can be used to reduce the influence of the distortion and aberration (see Figure 6(b)). From Figure 6(c), it is noted that although there is some sinusoidal fluctuation on the surface, the cuts in the pork liver are clearly presented in the reconstructed shape. Factors like the nonlinear response of the CCD camera and the projector should be responsible for the sinusoidal error which may be removed by improving the resolution, correcting the gray level and increasing the 
number of the frequency used.

\section{Conclusion}

In this paper, a five-step phase shifting method based fringe projection profilometry with multiple frequencies applying to the measurement of biological tissues is described. By adopting the peak searching algorithm which independently calculates the points on the object, the phase unwrapping process is avoided and this method is suitable to the measurement of the objects with large steps. The final results suggest that the technique mentioned may have great potential in the field of the biology and medical science.

\section{Acknowledgments}

This work is supported by the Science Foundation of Shaanxi Province (2014K07-02) and National High Technology Research and Development Program of China (863 Program 2015AA020303).

\section{References}

[1] D.L. Burk, D.C. Mears, W.H. Kennedy, L.A. Cooperstein and D.L. Herbert, Three-dimensional computed tomography of acetabula fractures, Radiology 155 (1985), 183-186.

[2] D.C. Hemmy, D.J. David and G.T. Herman, Three-dimensional reconstruction of craniofacial deformity using computed tomography, Neurosurgery 13 (1983), 534-541.

[3] D.C. Hemmy and P.L. Tessier, CT of dry skulls with craniofacial deformities: Accuracy of three dimensional reconstruction, Radiology 157 (1985), 113-116.

[4] William E. Lorensen and Harvey E. Cline, Marching cubes: A high resolution 3D surface construction algorithm, Computer Graphics 21 (1987), 163-169.

[5] Wei Sun and Paliavi Lai, Recent development on computer aided tissue engineering-a review, Computer Methods and Programs in Biomedicine 67 (2002), 86-103.

[6] H.S. Baik, J.M. Jeon and H.J. Lee, Facial soft-tissue analysis of Korean adults with normal occlusion using a 3-dimensional laser scanner, American Journal of Orthodontics and Dentofacial Orthopedics 131 (2007), 759-766.

[7] F. Chen, G.M. Brown and M. song, Overview of the three dimensional shape measurement using optical method, Optical Engineering 39 (2000), 10-22.

[8] C. Runte, D. Dirksen, H. Delere, et al., Optical data acquisition for computer-assisted design of facial prostheses, The International Journal of Prosthodontics 15 (2002), 129-132

[9] Yao-yang Xiong, Ting Jiao and Fu-qiang Zhang, Application of three-dimensional structured-light measurement technique and rapid prototyping technique in maxillofacial prosthetics, Journal of Clinical Rehabilitative Tissue Engineering Research 12 (2008), 1705-1708.

[10] X.-Y. Su, W.-S. Zhou, G. von Bally and D. Vukicevic, Automated phase-measuring profilometry using defocused projection of a Ronchi grating, Optics Communications 94 (1992), 561-573.

[11] H. Zhang, M.J. Lalor and D.R. Burton, Spatiotemporal phase unwrapping for the measurement of discontinuous objects in dynamic fringe-projection phase-shifting profilometry, Applied Optics 38 (1999), 3534-3541.

[12] X.Y. Su and W. Chen, Fourier transform profilometry: A review, Optics and Lasers in Engineering 35 (2001), 263-284.

[13] V. Srinivasan, H.C. Liu and M. Halioua, Automated phase-measuring profilometry of 3-D diffuse objects, Applied Optics 23 (1983), 3105-3108.

[14] X. Su and W. Chen, Reliability-guided phase unwrapping algorithm: A review, Optics and Lasers in Engineering 42 (2004), 245-261.

[15] D.C. Ghiglia and L.A. Romero, Robust two-dimensional weighted and unweighted phase unwrapping that uses fast transforms and iterative methods, Journal of the Optical Society of America A 11 (1994), 107-117. 
[16] C.A. Hobson, H.T. Atkinson and F. Lilley, The application of digital filtering to phase recovery when surface contouring using fringe projection techniques, Optics and Lasers in Engineering 27 (1997), 355-368.

[17] Libing Zhou, Xianyu Su and Liwu Wang, Analysis of errors introduced by detector nonlinearity in Phase Measuring Profilometry, Laser Journal 23 (2002), 19-21.

[18] Yong Xu, Shuhai Jia, Qingchen Bao, Hualing Chen and Jia Yang, Recovery of absolute height from wrapped phase maps for fringe projection profilometry, Optics Express 22 (2014), 16819-16828. 